\title{
Spoken-Word Recognition: The Access to Embedded Words
}

\author{
Frédéric Isel \\ Max Planck Institute of Cognitive Neuroscience, Leipzig, Germany \\ and \\ Nicole Bacri
}

Laboratoire de Psychologie Expérimentale, Université de Paris V-Centre National de la Recherche Scientifique, Paris, France

Two cross-modal priming experiments investigated whether the representation of either an initial- or a final-embedded word may be activated when the longer carrier word is auditorily presented. Visual targets were semantically related either to the embedded word or to the carrier word or they were unrelated to the primes. A priming effect was found for semantic associates of the carrier word in both experiments as well as for associates of the final-embedded word (Experiment 2). These results suggest that mapping speech onto lexical representations may imply lexical access attempts at several points during auditory processing of the speech signal. () 1999 Academic Press

Key Words: embedded words; word recognition; cross-modal semantic priming.

Lexical access requires finding a phonologically legal match between acoustic-phonetic information and representations stored in the mental lexicon (Gaskell \& Marslen-Wilson, 1996). Although the speech signal is segmented during processing, very few reliable acoustic cues concerning the boundaries of real words are present during continuous speech. The question we addressed was whether lexical access is triggered only by the acousticphonetic onset of an auditory sequence, which implies an initial alignment

This research was supported by a grant from the Fondation Fyssen (France) to F. Isel. We thank Angela Friederici and Juan Segui for their fruitful comments on a previous version of this article.

Part of this research was reported at the XXIIèmes Journées d'Etudes sur la Parole, Martigny, Suisse, June, 1998.

Address correspondence and reprint requests to Frédéric Isel, Max Planck Institute of Cognitive Neuroscience, Stephanstrasse 1a, Postfach 500 355, D-04103 Leipzig, Germany. Fax: +49(0) (341) 9940-113. E-mail: isel@cns.mpg.de.

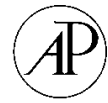


(i.e., only the first two phonemes trigger lexical access attempts) as proposed by the Cohort model (Marslen-Wilson, 1987), or whether lexical access can be activated continuously and at each point during the signal, which thus implies an exhaustive alignment as proposed by the TRACE model of McClelland and Elman (1986). One important implication of this account is that lexical competition between two simultaneously activated candidates may be expected when those two candidates consist of a carrier word and a short word embedded within that carrier word.

We used as linguistic materials disyllabic words within which an embedded word was located either initially or finally. For example, the French words cargo ("freighter") and brancard ("stretcher') contain a monosyllabic word, car ("bus"), embedded either in an initial or in a noninitial position. In order to examine whether the activation level of lexical candidates depends on the location of their acoustic onset, we used cross-modal semantic priming within a lexical decision task. For this task, the auditory primes were composed of disyllabic carrier words and the visual targets were either semantically related to the embedded word or to the carrier word. Subjects had to make a lexical decision task on the visual targets.

According to the Cohort model, initial-embedded words receive a transient activation during speech coding, whereas final-embedded words are not activated (Marslen-Wilson \& Zwitserlood, 1989). Thus, an initial-embedded word is a part of a set of word candidates for recognition, whereas finalembedded words are not present within the cohort set. According to TRACE, several lexical candidates, beginning at different points along the speech signal, can be activated simultaneously, even if the best matching candidate (usually the longest word) presents the highest activation level. Gow and Gordon (1995) confirmed that long words exhibit an advantage for selection, but also showed that shorter words can be accessed. In addition, simulations have demonstrated the residual activation of embedded words (e.g., "car", embedded in "carpet"; see Frauenfelder \& Peeters, 1990). Shillcock (1990) found a facilitatory priming effect for the visual target RIB preceded by the auditory prime "trombone" (i.e., "bone" facilitated the processing of RIB). In addition, facilitatory priming effects have been observed for targets semantically related to the final-position embedded words, e.g., boos (meaning " "angry") in framboos ("raspberry") facilitated the processing of KWAAD (meaning " "angry" as well) (Vroomen \& de Gelder, 1997). Moreover, Vroomen and de Gelder showed the presence of a residual activation of short words initial-embedded in longer pseudowords. In order to establish an experimental basis for these model-based accounts of lexical priming, the effect of the location of the embedded word on its activation level was studied in two experiments. In Experiment 1, the embedded word corresponded to the initial syllable of carrier words. In Experiment 2, the same embedded words corresponded to the final syllable of the disyllabic carriers. 
TABLE 1

Sample Stimulus Set

\begin{tabular}{lcc}
\hline Visual targets & Related auditory primes & Unrelated auditory primes \\
\hline bateau $^{a}$ & cargo & sulfate $^{d}$ \\
autobus $^{b}$ & cargo & sulfate \\
\hline
\end{tabular}

a "Boat," related to the carrier word "cargo."

$b$ "'Bus," related to the embedded word "car" ("bus").

c "'Freighter," experimental auditory prime.

$d$ "'sulfate," control auditory prime.

\section{EXPERIMENT 1}

Experiment 1 was designed to study the activation level of monosyllabic words embedded in the initial position (i.e., initial-embedded words) of disyllabic carrier words. If the embedded words are processed and if their activation is maintained until the acoustic offset of the carrier sequence, facilitatory priming effects for the embedded words were expected. In previous experiments, we found priming effects when initial-embedded words were phonologically related to their primes (Isel \& Bacri, submitted). Alternatively, a long-word advantage resulting from an inhibition of the embedded words by the longer carriers should suppress all priming effects.

\section{Method}

Subjects. Forty undergraduate students at the Université René Descartes, Paris, participated in a single 20-min session. All were native Parisian French speakers with no reported auditory or (uncorrected) visual deficits.

Materials. In the experimental condition, 36 disyllabic monomorphemic words (mean duration: $520 \mathrm{~ms}$ ) whose first syllables were words (mean duration: $203 \mathrm{~ms}$ ) were used as auditory primes. Seventy-two di- and trisyllabic words (mean, 6.2 letters) were used as visual targets of which 36 were semantically related to the disyllabic primes and 36 were related to the monosyllabic words embedded in these primes. A pilot, semantic association experiment conducted with 20 subjects determined the best associated targets. In the control condition, the same 72 visual targets were used but they were preceded by phonologically and semantically unrelated disyllabic words. One hundred and eight filler pairs were added (36 word-word pairs, and 72 word-pseudoword pairs). All words were middle-frequency nouns. A sample set is presented in Table 1.

Design and procedure. Each trial consisted of an auditory prime and a visual target. Four lists were created such that related and unrelated pairs were balanced across lists. Each target was presented under two priming conditions, but no subject heard the same prime twice or saw the same target twice. In each list, 18 related pairs and 18 unrelated pairs were presented. The proportion of related pairs was $12.5 \%$ to prevent subjects from adopting a guessing strategy based on the detection of semantic relations in the materials. Auditory primes were recorded by a French male speaker without salient $F 0$ changes. Subjects were instructed to indicate, as accurately and quickly as possible, whether visual targets were words or nonwords. The RTs were recorded for each trial from the acoustic offset of the auditory carrier words. The interstimulus interval was set at $0 \mathrm{~ms}$ and the intertrial interval was $2 \mathrm{~s}$. 


\section{TABLE 2}

Experiment 1: Mean Lexical Decision Times and Standard Deviations (in Milliseconds) for Targets Associated to the Mono- and Disyllabic Primes in Related and Unrelated Conditions

\begin{tabular}{lcc}
\hline Condition & Monosyllable-related targets & Disyllable-related targets \\
\hline Related & $536(35)$ & $502(30)$ \\
Unrelated & $537(37)$ & $538(36)$ \\
Priming effect & +1 & +36 \\
\hline
\end{tabular}

\section{Results and Discussion}

Table 2 displays the mean RTs for each priming condition. Analyses of variance (ANOVAs) across subjects $(F 1)$ and items $(F 2)$ were performed on RTs with Condition (related, unrelated) and Target Type (related to the embedded word or to the carrier word) as within-subjects factors. Mean lexical decision latency was faster for the related condition $(519 \mathrm{~ms}, S D=28$ $\mathrm{ms})$ than for the unrelated condition $(537 \mathrm{~ms}, S D=30 \mathrm{~ms})[F 1(1,39)=$ $17.4, p<.0001 ; F 2(1,35)=15.6, p<.0004]$. Targets related to the embedded words (537 ms, $S D=32 \mathrm{~ms}$ ) were processed significantly slower than targets related to the carrier words $(520 \mathrm{~ms}, S D=28 \mathrm{~ms})[F 1(1,39)=11.6$, $p<.001 ; \mathrm{F} 2(1,35)=6.8, p<.04]$. The interaction between Condition and Target Type was significant $[F 1(1,39)=21.3, p<.0001 ; F 2(1,35)=8.6$, $p<.005]$. A significant facilitatory priming effect was observed only for the targets associated to the carrier words $(36 \mathrm{~ms})[F(1,39)=36, p<.0001]$.

The results of Experiment 1 clearly show that no residual activation of semantic forms associated to the embedded words was present at the acoustic offset of the carrier words. Initial alignment of two words at the acoustic onset of an auditory sequence is not sufficient to activate semantic associates of the initial-embedded word. However, the data do not support the conclusion that short embedded words were not processed. Indeed, the question of whether these words were effectively processed but their activation too transient to be measured still needed to be addressed.

\section{EXPERIMENT 2}

The goal of Experiment 2 was to test for activation associated with the embedded words. In order to increase the possibility of detecting this activation, embedded words were located on the final syllable of disyllabic primes. The embedded-word set was the same as in Experiment 1, e.g., brancard ("stretcher") contained car ("bus") as did cargo ("freighter"). It was hypothesized that, if embedded words are processed, the level of activation will be measured at their acoustic offset and this would allow determination of whether access to these words has been attempted. The Cohort model predicts no lexical access attempts for words that are not in the cohort of 
TABLE 3

Sample Stimulus Set

\begin{tabular}{lcc}
\hline Visual targets & Related auditory primes & Unrelated auditory primes \\
\hline hôpital $^{a}$ & $\begin{array}{l}\text { brancard }^{c} \\
\text { brancard }\end{array}$ & $\begin{array}{l}\text { sulfate }^{d} \\
\text { autobus }\end{array}$
\end{tabular}

a "Hospital," related to the carrier word "brancard.",

$b$ "'Bus,", related to the embedded word "car" ("bus").

c "'Stretcher," experimental auditory prime.

$d$ "'Sulfate," control auditory prime.

candidates, whereas models that involve the parallel processing of several candidates suggest that final-embedded words could be activated.

\section{Method}

The method was the same as in Experiment 1, with the following exceptions. Auditory primes consisted of 24 disyllabic words whose final syllables were analogous to the initial syllables of the primes used in Experiment 1 (see Table 3). According to the experiment, embedded words were acoustically different, due to coarticulation differences. However, they exhibited the same duration. The mean duration of the disyllabic words was $515 \mathrm{~ms}$ (embedded words: $215 \mathrm{~ms}$ ). In order to test for standardization of the stimulus materials used in Experiments 1 and 2, a $t$ test was performed on the duration of embedded words in the initial position (Experiment 1) vs the final position (Experiment 2) and no significant difference was observed.

\section{Results and Discussion}

Table 4 displays the mean RTs for each priming condition. An ANOVA revealed that targets were processed significantly faster in the related condition $(524 \mathrm{~ms}, S D=28 \mathrm{~ms})$ than in the unrelated condition $(562 \mathrm{~ms}, S D=$ $39 \mathrm{~ms})[F 1(1,39)=43, p<.00001) ; F 2(1,23)=51, p<.00001]$. The main effect of Target Type was not significant across either subjects or items. The interaction between Condition and Target Type was significant $[F 1(1$, $39)=4.5, p<.04) ; F 2(1,35)=4.5, p<.04]$. Priming effects were significant for the targets related to the disyllabic primes $(+48 \mathrm{~ms} ; F(1,39)=$ $38.4, p<.00001]$ and for the targets related to the embedded words $(+27$ $\mathrm{ms} ; F(1,39)=4.5, p<.04]$.

\section{TABLE 4}

Experiment 2: Mean Lexical Decision Times and Standard Deviations (in Milliseconds) for Targets Associated to the Mono- and Disyllabic Primes in Related and Unrelated Conditions

\section{Condition}

Related

Unrelated

Priming effect
Monosyllable-related targets

Disyllable-related targets

$+48$ 
These results showed that the final-embedded words were significantly processed, although priming effects were weaker for targets associated with embedded words than for targets associated with carrier words.

\section{GENERAL DISCUSSION}

This study aimed to investigate whether lexical access attempts are performed only at the acoustic onset of an auditory sequence or at several points during this sequence. The results of two experiments showed that words embedded in the final position of longer words are processed, whereas initialembedded words are not sufficiently activated to give rise to priming effects. In other words, it was revealed that words whose acoustic onsets do not match the acoustic onsets of the carriers in which they are embedded can be accessed. A comparison of Experiments 1 and 2 suggests that the absence of priming effects observed in Experiment 1 was due to the "deactivation", of semantic forms associated with embedded words and was not due to the absence of lexical processing for these words. Thus, it can be assumed that lexical access may be generated in at least two points during signal processing: at the beginning of the signal and at a median point. These findings support the notion of multiple alignment as proposed by TRACE, which seems a more realistic account than the initial alignment account proposed by Cohort. However, the data also suggest that a partial match between acoustic information and lexical representation results in a relatively fast decay of activation. The results of Experiment 1 suggested that the life-duration of the activation of semantic forms could be less than $250 \mathrm{~ms}$. Further experiments are necessary in order to determine the precise temporal functions associated with activation and deactivation and the qualitative nature of the deactivation process.

\section{REFERENCES}

Frauenfelder, U. H., \& Peeters, G. 1990. On lexical segmentation in TRACE: An exercise in simulation. In G. Altmann (Eds.), Cognitive models of speech processing: Psycholinguistic and computational perspectives (pp. 50-86). Cambridge, MA: MIT Press.

Gaskell, G., \& Marslen-Wilson, W. D. 1996. Phonological variation and inference in lexical access. Journal of Experimental Psychology: Human Perception and Performance, 22, $144-158$.

Gow, D. W., \& Gordon, P. C. 1995. Lexical and prelexical influences on word segmentation: Evidence from priming. Journal of Experimental Psychology: Human Perception and Performance, 21, 344-359.

Isel, F. \& Bacri, N. Submitted. Lexical competition and the processing of embedded words: A cross-modal repetition priming study. Quarterly Journal of Experimental Psychology.

Marslen-Wilson, W. D. 1987. Functional parallelism in spoken word recognition. Cognition, 25, 71-102.

Marslen-Wilson, W. D., \& Zwitserlood, P. 1989. Accessing spoken words: The importance 
of word onsets. Journal of Experimental Psychology: Human Perception and Performance, 15, 576-585.

McClelland, J. L., \& Elman, J. L. 1986. The TRACE model of speech perception. Cognitive Psychology, 18, 1-86.

Shillcock, R. 1990. Lexical hypotheses in continuous speech. In G. Altmann (Ed.), Cognitive models of speech processing: Psycholinguistic and computational perspectives (pp. 2449). Cambridge, MA: MIT Press.

Vroomen, J., \& de Gelder, B. 1997. The activation of embedded words in spoken word recognition. Journal of Experimental Psychology: Human Perception and Performance, 23, 710720 . 